\title{
The Catastrophe Map of a Two Period Production Model with Uncertainty
}

\author{
Pascal Stiefenhofer ${ }^{1,2}$ \\ ${ }^{1}$ Department of Statistics, University College London, London, UK \\ ${ }^{2}$ Department of Mathematics, University of Sussex, East Sussex, UK \\ Email: p.stiefenhofer@ucl.ac.uk
}

Received June 5, 2013; revised July 5, 2013; accepted July 12, 2013

Copyright (C) 2013 Pascal Stiefenhofer. This is an open access article distributed under the Creative Commons Attribution License, which permits unrestricted use, distribution, and reproduction in any medium, provided the original work is properly cited.

\begin{abstract}
This paper shows existence and efficiency of equilibria of a two period production model with uncertainty as a consequence of the catastrophe map being smooth and proper. Its inverse mapping defines a finite covering implying finiteness of equilibria. Beyond the extraction of local equilibrium information of the model, the catastrophe map renders itself well for a global study of the equilibrium set. It is shown that the equilibrium set has the structure of a smooth submanifold of the Euclidean space which is diffeomorphic to the sphere implying connectedness, simple connectedness, and contractibility.
\end{abstract}

Keywords: Differential Topologyl; General Equilibrium; Uncertainty; Production

\section{Introduction}

This paper considers a two period production model with uncertainty. The time structure and associated uncertainty is described by a finite number of uncertain states of the world. It is assumed that all firms are owned by the consumers according to an exogenously determined ownership structure. This economic scenario describes the private ownership model discussed in Debreu [1] where the objective of each firm is to maximize profits. The seminal paper of this model without uncertainty dates back to the path breaking paper by Arrow and Debreu [2].

In this paper, we show that many economically interesting equilibrium properties of the two period production model with uncertainty can be derived from the catastrophe map. For that purpose we follow the mathematical approach discussed in Balasko [3] and in Dierker [4].

More specifically, we describe the set of solutions of all two period production economies and explore its structure. It is shown that this set is a smooth submanifold of the Euclidean space which is diffeomorphic to the sphere. A study of some of the properties of the catastrophe map enables us to characterize the set of economies into sets with various properties, such as economies with singular equilibria, economies with multiple equilibria, and economies with catastrophes, where equilibrium behavior is more difficult to study. Most of these properties have been studied in the context of exchange economies [5] or simple production economies [6-10] or Balasko (Preprint 2011) for example ${ }^{1}$. This paper generalizes the economic scenario by adding more structure to the model of the firm and thus moving towards a more realistic model where time and uncertainty is present.

The structure of the paper is as follows: Section 1 is an introduction. Section 2 introduces the economic scenario and states a definition of economic equilibrium. Section 3 explores the topological structure of the equilibrium set of all two period production economies with uncertainty. The next section states equilibrium properties of the model such as existence, efficiency and finiteness of equilibria. The final section is a conclusion.

\section{The Long Run Private Ownership Production Model with Uncertainty}

We describe the two period private ownership production model $\mathcal{P}(L)$ introduced in Debreu ([1], chapter 7). Uncertainty is defined by a finite set of mutually exclusive and exhaustive states of nature denoted by $s \in\{0,1, \cdots, S\}$, where $s=0$ is the certain event in time period one and $\{1,2, \cdots, S\}$ are the uncertain events in time period two. In total there are $S+1$ states of nature. There are $i \in\{1, \cdots, m\}$ consumers, $j \in\{1, \cdots, n\}$ producers, and $k \in\{1, \cdots, l\}$ physical goods. For all consumers $i \in\{1, \cdots, m\}$, a consumption bundle is a collection of

${ }^{1}$ Discussion paper: The natural projection approach to smooth production economies, 2011. 
vectors $x_{i}=\left(x_{i}(0), \cdots, x_{i}(s), \cdots, x_{i}(S)\right) \in X_{i}=\mathbb{R}_{++}^{l(S+1)}$, where consumption in a particular state $s \in\{0,1, \cdots, S\}$ is a vector $x_{i}(s)=\left(x_{i}^{1}(s), \cdots, x_{i}^{l}(s)\right) \in \mathbb{R}_{++}^{l}$. Associated with physical commodities is a set of normalized prices, denoted $\mathbf{S}=\left\{p \in \mathbb{R}_{++}^{l(S+1)}: p^{l}(s)=1, \forall s \in\{0,1, \cdots, S\}\right\}$.

Consumers are further endowed with a fraction $\theta_{i j} \in[0,1]$ of the profits of each firm. $\theta_{i j}$ represents the exogenously determined ownership structure of the private ownership production economy. It satisfies for each $j \in\{1, \cdots, n\}$ and $i \in\{1, \cdots, m\}, 0 \leq \theta_{i j} \leq 1$, and $\sum_{i} \theta_{i j}=1$. Denote the set of ownership structures

$\Theta=\left\{\theta_{i j} \in \mathbb{R}_{+}^{n m}: \sum_{i} \theta_{i j}=1, \forall j \in\{1, \cdots, n\}\right\}$.

Consumers are endowed with a collection of vectors of initial resources denoted by

$\omega_{i}=\left(\omega_{i}(0), \cdots, \omega_{i}(s), \cdots, \omega_{i}(S)\right) \in \Omega_{i}=\mathbb{R}_{++}^{l(S+1)}$, where initial endowments in a particular state $s \in\{0,1, \cdots, S\}$ is a vector $\omega_{i}(s)=\left(\omega_{i}^{1}(s), \cdots, \omega_{i}^{l}(s)\right) \in \mathbb{R}_{++}^{l}$. Consumer $i \in\{1, \cdots, m\}$ is further characterized by a smooth Marschallian demand function $f_{i}: \mathbf{S} \times \mathbb{R}_{++}^{S+1} \rightarrow \mathbb{R}_{++}^{l(S+1)}$, where $f_{i}\left(p, w_{i}\right)$ is defined for price vector $p \in \mathbf{S}$ and wealth level $w_{i} \in \mathbb{R}_{++}^{S+1},[11]$, where $w_{i}(s)=p(s) \cdot \omega_{i}(s)$ for all $s \in\{0,1, \cdots, S\}$.

Producers are characterized by production sets and their smooth supply functions. The main property of the long run production model is that all activities of the firm are variable. An activity $y_{j}$ is a collection of vectors $y_{j}=\left(y_{j}(0), \cdots, y_{j}(s), \cdots, y_{j}(S)\right) \in \mathbb{R}^{l(S+1)}$, where an activity in state $s=0$ is a vector of inputs

$y_{j}(0)=\left(y_{j}^{1}(0), \cdots, y_{j}^{l}(0)\right) \in \mathbb{R}_{-}^{l}$, and

$y_{j}(s)=\left(y_{j}^{1}(s), \cdots, y_{j}^{l}(s)\right) \in \mathbb{R}_{-}^{l}$ is the associated vector of outputs in state $s \in\{1, \cdots, S\}$. Let $\xi_{j}: \mathbf{S} \rightarrow \mathbb{R}^{l(S+1)}$ denote the smooth supply function of firm $j \in\{1, \cdots, n\}$, where $\xi_{j}(p)$ is defined on the set of normalized prices. Standard assumptions of smooth production economies introduced in [1] hold for each production set $Y_{j} \subset \mathbb{R}^{l(S+1)}$. In particular $Y_{j}$ is convex, $0 \in Y_{j}$, and $\partial Y_{j}$ has a strictly positive Gaussian curvature for every $j \in\{1, \cdots, n\}$. These assumptions imply that supply functions are smooth.

\section{Equilibrium $\mathcal{P}(L)$}

Each consumer $i \in\{1, \cdots, m\}$ chooses a utility maximizing consumption bundle $x_{i} \in X_{i}$ at fixed $\omega_{i} \in \Omega$ and $\theta_{i j} \in \Theta$ satisfying his budget constraints. Each producer $j \in\{1, \cdots, n\}$ chooses profit maximizing net activities $y_{j} \in Y_{j}$ at competitive prices $p \in \mathbf{S}$. Let

$$
\begin{aligned}
& z(p(s), \omega(s), \theta(s)): \\
= & \sum_{i}^{m} f_{i}\left(p(s), p(s) \cdot \omega_{i}(s)+\sum_{j}^{n} \theta_{i j}(s) p(s) \cdot \xi_{j}(p(s))\right)(1) \\
& -\sum_{i}^{m} \omega_{i}(s)+\sum_{j}^{n} \xi_{j}(p(s))
\end{aligned}
$$

be the market excess demand function in state $s \in\{0,1, \cdots, S\}$. Then, market clearance requires demand to equal supply in each market and uncertain state of the world. Hence

$$
z(p(s), \omega(s), \theta(s))=0, \forall s \in\{0,1, \cdots, S\} .
$$

An equilibrium is a price vector $p \in \mathbf{S}$ which satisfies this equation for a fixed distribution of initial resources and exogenously given ownership structure. An equilibrium pair is an equilibrium price vector $p \in \mathbf{S}$ with associated $\omega \in \Omega$. An equilibrium allocation is an allocation $(x, y, \theta)$ associated with an equilibrium price $p \in \mathbf{S}$. The model of the consumer is to solve a constraint optimization problem. This requires a consumer to maximize utility subject to a sequence of $(S+1)$ budget constraints. Hence, each consumer $i \in\{1, \cdots, m\}$ $\left(x_{i}(s)\right) \in \arg \max \left\{u_{i}(x(s)): x_{i} \in B_{i}(s)\right\}, \forall s \in\{0,1, \cdots, S\}$,

where $u_{i}: \mathbb{R}^{l(S+1)} \rightarrow \mathbb{R}$ is the consumer's smooth ${ }^{2}$ utility function. The production adjusted consumer budget set is defined by

$$
\begin{aligned}
B_{i}(s):= & \left\{x_{i}(s) \in \mathbb{R}_{++}^{l}: p(s) \cdot x_{i}(s)\right. \\
& \left.=p(s) \cdot \omega_{i}(s)+\sum_{j=1}^{n} \theta_{i j}(s) p(s) \cdot y_{j}(s)\right\}
\end{aligned}
$$

The model of the producer is to maximize profits. Each producer solves a constraint optimization profit maximization problem. Hence, each $j \in\{1, \cdots, n\}$

$$
\begin{aligned}
& \left(y_{j}(s)\right) \in \arg \max \left\{p(s) \cdot y_{j}(s): y_{j}(s) \in Y_{j}(s)\right\}, \\
& \forall s \in\{0,1, \cdots, S\},
\end{aligned}
$$

where the state dependent production set $Y_{j}(s)$ for all $s \in\{0,1, \cdots, S\}$ satisfies the assumptions of Debreu [1].

Definition 1. An equilibrium of the two period private ownership production model with uncertainty $\mathcal{P}(L)$ is a price vector $p \in \mathbf{S}$ at fixed pair $(\omega, \theta) \in \Omega \times \Theta$ if for utility maximizing consumers $i \in\{1, \cdots, m\}$ and profit maximizing producers $j \in\{1, \cdots, n\}$

$$
z(p(s), \omega(s), \theta(s))=0, \text { for all } s \in\{0,1, \cdots, S\} .
$$

An equilibrium allocation is a pair $(x, y) \in \mathbb{R}_{++}^{l(S+1) m} \times \mathbb{R}^{l(S+1) n}$ associated with an equilibrium price vector $p \in \mathbf{S}$ for fixed parameters $(\omega, \theta) \in \Omega \times \Theta$. Let denote the mathematical operation defined by a state by state inner product. There are $l(S+1)$ equilibrium equations less $(S+1)$ equations satisfying Walras' law $p z(p, \omega)=0$, hence we have a system of $l(S+1)-(S+$ 1) linearly independent equations. This amounts to the number of unknowns, given the number of normalized prices of $(S+1)$.

\footnotetext{
2"Smoothness" follows from the assumptions stated in [11]. It essentially means that all functions are differentiable at any order required.
} 
A study of the qualitative equilibrium structure of the two period private ownership production model with uncertainty amounts to a study of the structure of the solution set of the equilibrium Equation (2).

\section{Equilibrium Structure $\mathcal{E}$ of the Model $\mathcal{P}(\boldsymbol{L})$}

Let $\mathcal{E} \subset \mathbf{S} \times \Omega$ denote the set of equilibrium solutions of the two period production model with uncertainty $\mathcal{P}(L)$. This set consists of pairs $(p(s), \omega(s)) \in \mathbf{S} \times \Omega$ satisfying the equilibrium equations $z(p(s), \omega(s))=0$ for all $s \in\{0,1, \cdots, S\}$. Formally, we have

$$
\begin{aligned}
\mathcal{E}:= & \{(p(s), \omega(s)) \in S \times \Omega: \\
& \sum_{i}^{m} f_{i}\left(p(s), p(s) \cdot \omega_{i}(s)+\sum_{j}^{n} \theta_{i j}(s) p(s) \cdot \xi_{j}(p(s))\right) \\
& \left.-\left(\sum_{i}^{m} \omega_{i}(s)+\sum_{j}^{n} \xi_{j}(p(s))\right)=0\right\}, \forall s \in\{0,1, \cdots, S\} .
\end{aligned}
$$

For the proof of the next theorem we need the following result.

Lemma 1 (Properness of a mapping). Suppose $M(s)$ is a compact space and $N(s)$ is a Hausdorff space for every $s \in\{0,1, \cdots, S\}$. Then every continuous map $f(s): M(s) \rightarrow N(s)$ for all $s \in\{0,1, \cdots, S\}$ is proper.

Proof. We need to show that for every compact set $B(s) \subset N(s)$ the inverse image $f^{-1}(B(s))$ is compact for every $s \in\{0,1, \cdots, S\}$.

1) Let us show that the direct image $\operatorname{Im} f(A(s))$ of any closed subset $A(s)$ of $M(s)$ is closed in $N(s)$ for all $s \in\{0,1, \cdots, S\}$. To show this let $y(s)=\lim _{n(s) \rightarrow \infty} y(s)_{n(s)}$, for all $s \in\{0,1, \cdots, S\}$, where $\left\{y(s)_{n(s)}\right\}$ belongs to the set $f(A(s))$. From the convergence property of the sequence $\left\{y(s)_{n(s)}\right\}$ we see that the set $B(s)=\left\{\{y(s)\} \cup\left\{\bigcup_{n(s)}\left\{y(s)_{n(s)}\right\}\right\}\right\}$ is compact. From that it follows that $f^{-1}(B(s))$ is compact for every $s \in\{0,1, \cdots, S\}$.

2) Let us show that inverse image $\operatorname{Im} f^{-1}(B(s))$ is compact. We take $x(s)_{n(s)}$ in $A(s)$ such that $y(s)_{n(s)}=f\left(x(s)_{n(s)}\right)$. Clearly, the sequence $\left\{x(s)_{n(s)}\right\}$ belongs to the compact set defined by the inverse image $f^{-1}(B(s))$. Therefore, there exists a subsequence $\left\{x(s)_{n(s)_{r(s)}}\right\}$ for all $s \in\{0,1, \cdots, S\}$ such that $\lim _{r(s) \rightarrow \infty} x(s)_{n(s)_{r(s)}}=x(s) \quad([12]$, p. 41), where $x(s) \in f^{-1}(B(s))$. Since $x(s)$ is the limit of a subsequence of elements belonging to $A(s)$, we have $x(s) \in A(s)$. By continuity of the mapping $f$ we have $f(x(s))=\lim _{n(s) \rightarrow \infty} f(x(s))=y(s)=\lim _{n(s) \rightarrow \infty} y(s)_{n(s)}$.

This proves that $y(s) \in f(A(s))$ for every $s \in\{0,1, \cdots, S\}$.

Theorem 1. The set $\mathcal{E}$ of model $\mathcal{P}(L)$ is a closed subset of the Euclidean space defined by $\mathbf{S} \times \Omega$.

Proof. Note that continuity of the mapping

$$
\begin{aligned}
& (p(s), \omega(s)) \mapsto \\
& \sum_{i} f_{i}\left(p(s), p(s) \cdot \omega_{i}(s)+\sum_{j} \theta_{i j}(s) p(s) \cdot \xi_{j}(p(s))\right) \\
& -\left(\sum_{i} \omega_{i}(s)+\sum_{j} \xi_{j}(p(s))\right)=0
\end{aligned}
$$

for all $s \in\{0,1, \cdots, S\}$ is sufficient to show closedness of the set $\mathcal{E}$ of model $\mathcal{P}(L) . \mathcal{E}$ is the preimage of the vector $0 \in \mathbb{R}^{l(S+1)}$ by the smooth mapping

$$
\begin{aligned}
& (p(s), \omega(s)) \mapsto \\
& \sum_{i} f_{i}\left(p(s), p(s) \cdot \omega_{i}(s)+\sum_{j} \theta_{i j}(s) p(s) \cdot \xi_{j}(p(s))\right) \\
& -\left(\sum_{i} \omega_{i}(s)+\sum_{j} \xi_{j}(p(s))\right)=0
\end{aligned}
$$

for all $s \in\{0,1, \cdots, S\}$ which is closed by Lemma 1 . Continuity of the equilibrium equation is satisfied by the assumptions of differentiability of demand and supply mappings [1,11].

Theorem 2. The set $\mathcal{E}$ of model $\mathcal{P}(L)$ is a smooth manifold of dimension $(S+1) \mathrm{lm}$.

Proof. We consider the mapping $Z: \mathbf{S} \times \Omega \rightarrow \mathbb{R}^{l(S+1)}$ defined by the smooth mapping

$$
\begin{aligned}
& (p, \omega) \mapsto \sum_{i} f_{i}\left(p, p \cdot \omega_{i}+\sum_{j} \theta_{i j} p \cdot \xi_{j}(p)\right) \\
& -\left(\sum_{i} \omega_{i}+\sum_{j} \xi_{j}(p)\right)
\end{aligned}
$$

By the regular value theorem (Guillemin and Pollack [13], p. 21) $\mathcal{E}$ is the preimage of $0 \in \mathbb{R}^{l(S+1)}$. We need to prove that this mapping does not contain critical points. This follows by showing that the linear tangent map $D_{\omega} Z$ is onto. The onto property follows directly from the rank property of the Jacobian matrix chosen for any arbitrary individual $i \in\{1, \cdots, m\}$ and state of nature $s \in\{0,1, \cdots, S\}$. By the chain rule, we obtain

$$
=\left(\begin{array}{cccc}
\frac{\partial f_{i}^{1}(s)}{\partial \omega_{i}(s)} p^{1}(s)-1 & \cdots & \frac{\partial f_{i}^{1}(s)}{\partial \omega_{i}(s)} p^{l-1}(s) & \frac{\partial f_{i}^{1}(s)}{\partial \omega_{i}(s)} \\
\vdots & \ddots & \vdots & \vdots \\
\frac{\partial f_{i}^{l-1}(s)}{\partial \omega_{i}(s)} p^{1}(s) & \cdots & \frac{\partial f_{i}^{l-1}(s)}{\partial \omega_{i}(s)} p^{l-1}(s)-1 & \frac{\partial f_{i}^{l-1}(s)}{\partial \omega_{i}(s)}
\end{array}\right)
$$

By simple algebraic manipulations we obtain the new matrices 


$$
\begin{gathered}
\left(\begin{array}{cccc}
\frac{\partial f_{i}^{1}(s)}{\partial \omega_{i}(s)} p^{1}(s)-1-\frac{\partial f_{i}^{1}(s)}{\partial \omega_{i}(s)} p^{1}(s) & \cdots & \frac{\partial f_{i}^{1}(s)}{\partial \omega_{i}(s)} p^{l-1}(s) & \frac{\partial f_{i}^{1}(s)}{\partial \omega_{i}(s)} \\
\vdots & \ddots & \vdots & \vdots \\
\frac{\partial f_{i}^{l-1}(s)}{\partial \omega_{i}(s)} p^{1}(s)-\frac{\partial f_{i}^{1}(s)}{\partial \omega_{i}(s)} p^{1}(s) & \cdots & \frac{\partial f_{i}^{l-1}(s)}{\partial \omega_{i}(s)} p^{l-1}(s)-1 & \frac{\partial f_{i}^{l-1}(s)}{\partial \omega_{i}(s)}
\end{array}\right) \\
\left(\begin{array}{ccccc}
-1 & \cdots & \frac{\partial f_{i}^{1}(s)}{\partial \omega_{i}(s)} p^{l-1}(s)-\frac{\partial f_{i}^{1}(s)}{\partial \omega_{i}(s)} p^{l-1}(s) & \frac{\partial f_{i}^{1}(s)}{\partial \omega_{i}(s)} \\
\vdots & \ddots & \vdots & \vdots \\
0 & \cdots & \frac{\partial f_{i}^{l-1}(s)}{\partial \omega_{i}(s)} p^{l-1}(s)-1-\frac{\partial f_{i}^{1}(s)}{\partial \omega_{i}(s)} p^{l-1}(s) & \frac{\partial f_{i}^{l-1}(s)}{\partial \omega_{i}(s)}
\end{array}\right)
\end{gathered}
$$

Finally, we obtain

$$
\left(\begin{array}{cccc}
-1 & \cdots & 0 & \frac{\partial f_{i}^{1}(s)}{\partial \omega_{i}(s)} \\
\vdots & \ddots & \vdots & \vdots \\
0 & \cdots & -1 & \frac{\partial f_{i}^{l-1}(s)}{\partial \omega_{i}(s)}
\end{array}\right)
$$

from which we extract the information required. Rank $D_{\omega_{i}} Z$ is equal to $(l-1)$ in every state $s \in\{0,1, \cdots, S\}$. By the regular value theorem ([13], p. 21) $\mathcal{E}$ is a smooth manifold. This manifold is parameterized by smooth coordinate functions $\omega=(\omega(0), \cdots, \omega(s), \cdots, \omega(S)) \in \Omega$. From the regular value theorem it also follows that its dimension is equal to the dimension of $\mathbf{S} \times \Omega$ minus $(l-1)(S+1)$, hence

$$
\begin{aligned}
& \operatorname{dim}(\mathcal{E})=((l-1)(S+1)+m l(S+1)-(l-1)(S+1)) \\
& =m l(S+1)
\end{aligned}
$$

The following theorem illustrates a further economically interesting global property of the equilibrium manifold. It says that by construction of a diffeomorphism $f$ restricted to the equilibrium manifold $\mathcal{E}$ into

$\mathbf{S} \times \mathbb{R}_{++}^{m(S+1)} \times \mathbb{R}_{++}^{(l-1)(m-1)(S+1)} \quad \mathcal{E}$ is diffeomorphic to the sphere in $\mathbb{R}_{++}^{\operatorname{lm}(S+1)}$ implying that the equilibrium manifold is arc-connected, simply connected, and contractible. These properties are particularly useful in applied work such as economic policy equilibrium analysis. For example, economic policy is often concerned with finding a path between a current point on $\mathcal{E}$ and a desired point on $\mathcal{E}$. The following theorem proves that such a path always exists. In order to prove this result, we use a theorem given in (Hirsch [14], pp. 15-16).

Theorem 3. The smooth equilibrium manifold $\mathcal{E}$ of model $\mathcal{P}(L)$ is diffeomorphic to $\mathbb{R}_{++}^{l(S+1)}$.

Proof. The aim of the proof is to define two smooth mappings between smooth manifolds such that we can apply the theorem given in (Hirsch [14], pp. 15-16). Hence, let

$$
f: \mathbf{S} \times \Omega \rightarrow \mathbf{S} \times \mathbb{R}_{++}^{(S+1) m} \times \mathbb{R}_{++}^{(l-1)(S+1)(m-1)}
$$

be smooth mappings defined by

$$
\begin{aligned}
& f\left(p(s), \omega_{1}(s), \omega_{1}(s), \cdots, \omega_{m}(s)\right) \\
= & \left(p(s), p(s) \cdot \omega_{1}(s), p(s) \cdot \omega_{2}(s), \cdots, p(s) \cdot \omega_{m}(s),\right. \\
& \left.\bar{\omega}_{1}(s), \bar{\omega}_{2}(s), \cdots, \bar{\omega}_{m-1}(s)\right), \forall s \in\{0,1, \cdots, S\} .
\end{aligned}
$$

Then, let

$$
g: \mathbf{S} \times \mathbb{R}_{++}^{(S+1) m} \times \mathbb{R}_{++}^{(l-1)(S+1)(m-1)} \rightarrow \mathbf{S} \times \Omega
$$

denote smooth mappings defined by

$$
\begin{aligned}
& g\left(p(s), \bar{\omega}(s)_{1}, \omega_{1}^{l}(s), \cdots, \bar{\omega}_{m-1}(s), \omega_{m-1}^{l}(s), \bar{\omega}_{m}(s)\right), \\
& \forall s \in\{0,1, \cdots, S\} .
\end{aligned}
$$

Observe that the coordinates for the $l^{\text {th }}$ good of the $m-1$ consumers in $s \in\{0,1, \cdots, S\}, \omega_{1}^{l}(s), \cdots, \omega_{m-1}^{l}(s)$ are defined

$$
\begin{aligned}
& \omega_{i}^{l}(s)=w_{i}(s)-\left(\sum_{k=1}^{l-1} p^{k}(s) \cdot \omega_{i}^{l}(s)\right), \forall i \in\{1, \cdots, m-1\}, \\
& \forall k \in\{1, \cdots, l-1\} .
\end{aligned}
$$

Also observe that the coordinates for the $m^{\text {th }}$ consumer of the $l-1$ goods in $s \in\{0,1, \cdots, S\}$,

$$
\begin{aligned}
& \omega_{m}^{1}(s), \omega_{m}^{2}(s), \cdots, \omega_{m}^{l-1}(s) \text { are defined by } \\
& \omega_{m}(s)=\sum_{i=1}^{m} f_{i}\left(p(s), w_{i}(s)\right)-\sum_{i=1}^{m-1} \omega_{i}(s) .
\end{aligned}
$$

The application of the theorem in ([14]) requires to show that $\mathcal{E}=\operatorname{Im}(g)$ and that $f \circ g=I d$. The first part of the proof requires to calculate two inclusions, 1) $\operatorname{Im}(g) \subset \mathcal{E}$ and 2) $\mathcal{E} \subset \operatorname{Im}(g)$. We start by showing the second part first. Now, to show that 1$) \operatorname{Im}(g) \subset \mathcal{E}$, take any consumption bundle $x(s)=\left(p(s), w_{1}(s), \cdots, w_{m}(s), \bar{\omega}_{1}(s), \cdots, \bar{\omega}_{m-1}(s)\right)$, and 
compute the inner product of (4) with $p(s)$ $s \in\{0,1, \cdots, S\}$, and apply Walras' law to obtain

$$
w_{m}(s)=p(s) \cdot \omega_{m}(s), \forall s \in\{0,1, \cdots, S\} .
$$

From that a reformulation of (4) readily follows in terms of the production equilibrium equation

$$
\begin{aligned}
& \sum_{i} f_{i}\left(p(s), p(s) \cdot \omega_{i}(s)+\sum_{j} \theta_{i j}(s) p(s) \cdot \xi_{j}(p(s))\right) \\
& =\sum_{i} \omega_{i}(s)+\sum_{j} \xi_{j}(p(s)), \forall s \in\{0,1, \cdots, S\},
\end{aligned}
$$

hence $\operatorname{Im}(g) \subset \mathcal{E}$. Next, we need to show that 2) $\mathcal{E} \subset \operatorname{Im}(g)$. Take any arbitrary $(p(s), \omega(s)) \in \mathcal{E}$. It is then trivial to do the computations proving following equality

$$
f(s) \circ g(p(s), \omega(s))=(p(s), \omega(s)), \forall s \in\{0,1, \cdots, S\}
$$

from which it readily follows that $\mathcal{E} \subset \operatorname{Im}(g)$. Clearly we have constructed the two smooth relations such that

$$
f(s) \circ g(s)=I d(s), \forall s \in\{0,1, \cdots, S\},
$$

where $I d$ is the identity map defined on $\left(\mathbf{S} \times \mathbb{R}_{++}^{(S+1) m} \times \mathbb{R}_{++}^{(l-1)(S+1)(m-1)}\right)$. We have shown that the smooth mapping $f$ restricted to the equilibrium manifold $\mathcal{E}$ defines a diffeomorphism between $\mathcal{E}$ and the sphere of dimension $\mathbb{R}_{++}^{(S+1) l m}$.

\section{Existence, Efficiency, and Finiteness of Equilibria}

We now show that equilibria in the two period production model with uncertainty always exist. The strategy of the proof is to show that the catastrophe mapping $\pi: \mathcal{E} \rightarrow \Omega$ is smooth and proper. Existence of equilibria of this production model with uncertainty follows immediately from the smoothness proposition (1) and the properness proposition (2) below. The result of properness of $\pi$ provides a deep insight into the definition of economics itself. It implies that economic resources are scarce. The diffeomorphism $f_{i}\left(p(s), p(s) \cdot \omega_{i}(s)\right)$ for all $s \in\{0,1, \cdots, S\}$ between the spaces $\mathbf{S} \times \mathbb{R}^{S+1}$ and $\mathbb{R}^{l(S+1)}$ suggests that the vector

$x_{i}(s)=f_{i}\left(p(s), p(s) \cdot \omega_{i}(s)\right)$ tends to infinity in norm if prices tend to zero. It tends to zero if prices tend to infinity.

Axiom 4 (Bounded and strictly convex preferences). 1) The set of consumptions bundles indifferent or preferred to consumption bundle $x^{\prime}(s) \in \mathbb{R}_{++}^{l}$ for all $s \in\{0,1, \cdots, S\}$ is bounded from below for every $x(s) \in \mathbb{R}_{++}^{l}$ for all $s \in\{0,1, \cdots, S\}$. The preordering $\preceq_{i}$ is then said to be bounded from below; 2) The set of consumptions bundles indifferent or preferred to consumption bundle $x^{\prime}(s) \in \mathbb{R}_{++}^{l}$ for all $s \in\{0,1, \cdots, S\}$ is strictly convex for every $x(s) \in \mathbb{R}_{++}^{l}$ for all $s \in\{0,1, \cdots, S\}$. The preordering $\preceq_{i}$ is then said to be strictly convex.

Theorem 5. Equilibria of the two period production model with uncertainty $\mathcal{P}(L)$ always exist.

Definition 2. The catastrophe map $\pi$ is defined by the $\pi_{\text {proj }}: \mathcal{E} \rightarrow \Omega$. It is the restriction of the projection $(p, \omega) \rightarrow \omega$ of the set of equilibria $\mathcal{E} \subset \mathbf{S} \times \Omega$ into the space of economies $\Omega$.

Proposition 1 (Smoothness). $\pi: \mathcal{E} \rightarrow \Omega$ of model $\mathcal{P}(L)$ is smooth.

Proof. From Theorem (3) we know that $\mathcal{E}$ of model $\mathcal{P}(E)$ is a smooth submanifold of $\mathbf{S} \times \Omega$ which is diffeomorphic to the sphere of dimension $\mathbb{R}_{++}^{l m(S+1)}$. It follows from the definition of a smooth submanifold ([15], p. 174) that its natural embedding $\hat{\pi}: \mathcal{E} \rightarrow \mathbf{S} \times \Omega$ is smooth. It is clear that the projection mapping $\bar{\pi}: S \times \Omega \rightarrow \Omega$ is itself smooth. It then follows that $\pi$ the restriction of the natural projection to $\mathcal{E}$ as the composition of two smooth mappings $\pi=\bar{\pi} \circ \hat{\pi}$ is therefore smooth.

Proposition 2 (Properness). $\pi: \mathcal{E} \rightarrow \Omega$ of model $\mathcal{P}(L)$ is proper.

Proof. The strategy of the proof is to define the economic scenario such that lemma (1) can be applied to the model $\mathcal{P}(L)$. Hence, we need to show that for all $s \in\{0,1, \cdots, S\}$ the inverse image $\pi^{-1}(K(s))$, where $K(s)$ is a compact set in the space of initial resources, $\Omega(s)=\mathbb{R}_{++}^{l m}$, is compact.

We show that individual consumer demand is bounded below in every uncertain state of the world. To show this, consider any $i \in\{1,2, \cdots, m\}$ and define the projection of initial resource into the $i^{\text {th }}$ coordinate and state $s \in\{0,1, \cdots, S\}, \omega(s): \mathbb{R}_{++}^{l m} \rightarrow \mathbb{R}_{++}^{l}$ defined by

$$
\omega(s)=\left(\omega_{1}(s), \cdots, \omega_{m}(s)\right) \rightarrow \omega_{i}(s) .
$$

Pick an arbitrary $\omega_{i}(s)$ for $i \in\{1, \cdots, m\}$. Let $\omega_{i} \in K_{i}(s)$ be an element in a compact set $K_{i}(s)$. Note that $K_{i}(s)$ is compact by the projection $\omega(s)$ of a compact set $K(s)$ on the $i^{\text {th }}$ coordinate space. Compactness of $K_{i}(s)$ in $\Omega(s)_{i}$ implies for every $\omega_{i} \in K(s)_{i}$ that

$$
\omega_{i}^{\prime}(s) \leq \omega_{i}(s) \leq \omega_{i}^{\prime \prime}(s) .
$$

1) Now, for every $p(s) \in \mathbf{S}$ and $\omega_{i}(s) \in K_{i}(s)$ and $s \in\{0,1, \cdots, S\}$ need to show that $f_{i}\left(p(s), w_{i}(s)\right)$ is bounded from below. It then follows from standard assumptions of consumer theory that for all $s \in\{0,1, \cdots, S\}$

$$
u_{i}\left(\omega_{i}(s)\right) \leq u_{i}\left(f_{i}\left(p(s), w_{i}(s)\right)\right),
$$

where

$$
\begin{aligned}
& f_{i}\left(p(s), w_{i}(s)\right) \\
= & f_{i}\left(p(s), p(s) \cdot \omega_{i}(s)+\sum_{j}^{n} \theta_{i j}(s) p(s) \cdot \xi_{j}(p(s))\right),
\end{aligned}
$$


and $w_{i}(s)=p(s) \cdot \omega_{i}(s)+\sum_{j}^{n} \theta_{i j}(s) p(s) \cdot \xi_{j}(p(s))$

for all $s \in\{0,1, \cdots, S\}$.

By non satiation we also have

$$
u_{i}\left(\omega_{i}^{\prime}(s)\right) \leq u_{i}\left(\omega_{i}(s)\right),
$$

which by monotonicity of $u_{i}(s)$ implies that

$$
u_{i}\left(\omega_{i}^{\prime}(s)\right) \leq u_{i}\left(f_{i}\left(p(s), w_{i}(s)\right)\right) .
$$

Clearly, there exists some $x_{i}^{\prime}(s) \in \mathbb{R}_{++}^{l}$ for every $p(s) \in \mathbf{S}$ and $\omega_{i}(s) \in K_{i}(s)$ for all $s \in\{0,1, \cdots, S\}$ satisfying

$$
x_{i}^{\prime}(s) \leq u_{i}\left(f_{i}\left(p(s), w_{i}(s)\right)\right)
$$

by boundedness (Axiom 4) of indifference mappings from below for every $i \in\{1, \cdots, m\}$.

2) We now show that for every $p(s) \in \mathbf{S}$,

$\omega_{i}(s) \in K_{i}(s)$ and $s \in\{0,1, \cdots, S\}, h_{i}\left(p(s), w_{i}(s)\right)$ is also bounded from above. Consider the equilibrium price vector $p(s)$ for any $s \in\{0,1, \cdots, S\}$. Then for all pairs $\left(p(s), \omega_{i}(s)\right) \in \pi^{-1}(K(s))$ we have

$$
f_{i}\left(p(s), w_{i}(s)\right)=\sum_{i}^{m} \omega_{i}(s)-\sum_{-i}^{m} f_{i}\left(p(s), w_{i}(s)\right)
$$

where ${ }^{3}$

$$
\sum_{i}^{m} \omega_{i}(s)-\sum_{-i}^{m} f_{i}\left(p(s), w_{i}(s)\right) \leq \sum_{i}^{m} \omega_{i}(s)-\sum_{-i}^{m} x_{i}^{\prime}(s) .
$$

Clearly, $f_{i}\left(p(s), w_{i}(s)\right)$, is bounded above by some $x_{i}^{\prime \prime}(s) \in \mathbb{R}_{++}^{l}$, since for $(p(s), \omega(s)) \in \mathcal{E}, \quad \sum_{-i} \omega_{i}(s)$ is bounded from above for every $\omega(s) \in K(s)$. Hence, we have established the upper and lower bounds for every consumer $i \in\{1,2, \cdots, m\}$ given by

$$
\left\{x_{i}^{\prime}(s) \leq f_{i}\left(p(s), w_{i}(s)\right) \leq x_{i}^{\prime \prime}(s)\right\}
$$

for every $(p(s), \omega(s)) \in \pi^{-1}(K(s))$.

$3)$ We now apply Lemma 1 . For any arbitrary consumer $i \in\{1,2, \cdots, m\}$, we have established the compact set $\left\{x(s) \in \mathbb{R}_{++}^{l}: x_{i}^{\prime}(s) \leq x_{i}(s) \leq x_{i}^{\prime \prime}(s)\right\}, \forall s \in\{0,1, \cdots, S\}$. Let $G(s)$ be a compact set defined by the preimage of the diffeomorphism $h_{i}\left(p(s), w_{i}(s)\right)$ ([11]) projected onto $\mathbf{S}$. Hence, we observe that $\pi^{-1}(K(s))$ is a subset of the compact set $G(s) \times K(s)$. Lemma (1) requires to show that $\pi^{-1}(K(s))$ is closed in $G(s) \times K(s)$.

Now, by continuity of $\pi(s): \mathcal{E} \rightarrow \Omega(s)$, $\forall s \in\{0,1, \cdots, S\}$, it follows that $\pi^{-1}(K(s))$ is closed in $\mathcal{E}$, which by Theorem (1) is a closed subset of $\mathbf{S} \times \Omega$. Closedness of $\pi^{-1}(K(s))$ follows from closedness of $\pi^{-1}(K(s)) \cap G(s) \times K(s) \subset G(s) \times K(s)$.

Lemma 2 (Individual demand: Diffeomorphism of

\footnotetext{
${ }^{3}-i$ is standard notation used in economic theory. It is equivalent to saying $j \in\{1,2, \cdots, i, \cdots, m\}$ such that $i \neq j$, hence $\sum_{j \neq i}^{m}$.
}

$f_{i}$ ) For every $i \in\{1,2, \cdots, m\}$ the individual demand mapping $f_{i}: \mathbf{S} \times \mathbb{R}_{++}^{S+1} \rightarrow \mathbb{R}_{++}^{l(S+1)}$ is a diffeomorphism for all $s \in\{0,1, \cdots, S\}$.

Proof. The strategy of the proof is to show that $f_{i}\left(p(s), w_{i}(s)\right)$ is smooth, bijective, and that $f_{i}^{-1}$ is also smooth.

The problem of the consumer is to solve the constraint optimization given by

$$
\left(x_{i}(s)\right) \in \arg \max \left\{u_{i}(x(s)): x_{i} \in B_{i}(s)\right\}, \forall s \in\{0,1, \cdots, S\},
$$

where

$$
\begin{aligned}
B_{i}(s):= & \left\{x_{i}(s) \in \mathbb{R}_{++}^{l}: p(s) \cdot x_{i}(s)\right. \\
& \left.=p(s) \cdot \omega_{i}(s)+\sum_{j=1}^{n} \theta_{i j}(s) p(s) \cdot y_{j}(s)\right\} .
\end{aligned}
$$

We can use the Lagrangean method to solve this problem. Hence the solution of this problem satisfies the first order conditions of the optmimzation problem and is given by $x_{i}(s)=f_{i}\left(p(s), w_{i}(s)\right)$ for all $s \in\{0,1, \cdots, S\}$. Hence the pair $\left(x_{i}(s), \lambda(s)\right)$, where $\lambda(s)$ is the Lagrangian multiplier is a solution of the Lagrangian problem. Hence, to show smoothness of $f_{i}(s)$ requires to show that $\left(x_{i}(s), \lambda(s)\right)$ is a smooth function of $p(s)$ and $w_{i}(s)$. This is a consequence of the implicit function theorem applied to the solutions of the Lagrangian. Hence, we calculate the bordered Hessian matrix, $\bar{H}(s)$ for all $s \in\{0,1, \cdots, S\}$. Thus,

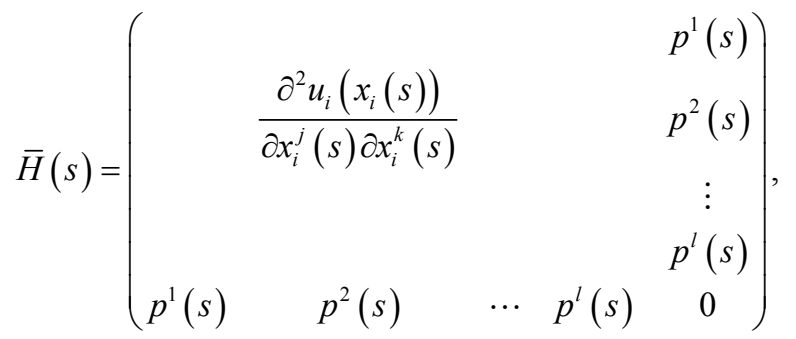

and the inverse of $\bar{H}(s)$ at $\left(x_{i}(s), \lambda(s), p(s), w_{i}(s)\right)$ exists since

$$
\operatorname{det} \bar{H}(s) \neq 0, \forall s \in\{0,1, \cdots, S\} .
$$

We now show that $f_{i}^{-1}$ is also smooth. Let $f_{i}^{-1}: \mathbb{R}_{++}^{l(S+1)} \rightarrow \mathbf{S} \times \Omega$ defined by

$$
g_{i}\left(x_{i}(s)\right)=\left(\nabla u_{i}\left(x_{i}(s)\right), x_{i}(s) \cdot \nabla u_{i}\left(x_{i}(s)\right)\right) .
$$

By assumptions of Debreu [11] all ingredients of this formula are smooth. Also the inner product of smooth functions is smooth. Hence we conclude that $f_{i}^{-1}$ is also smooth.

We now show that $f_{i}(s)$ and $g_{i}(s)$ are inverse mappings for all $s \in\{0,1, \cdots, S\}$. Hence

1) We calculate the individual composite mapping $f_{i}(s) \circ g_{i}(s)$ for all $s \in\{0,1, \cdots, S\}$ and show that $f_{i}(s) \circ g_{i}(s)=I d_{\mathbb{R}^{l(s+1)}}$. This condition is satisfied since 


$$
\begin{aligned}
f_{i}(s) \circ g_{i}(s) & =f_{i}\left(\nabla u_{i}\left(x_{i}(s)\right), x_{i}(s) \cdot \nabla u_{i}\left(x_{i}(s)\right)\right) \\
& =x_{i}(s), \forall s \in\{0,1, \cdots, S\} .
\end{aligned}
$$

As required, we have established $f_{i}(s) \circ g_{i}(s)=I d_{\mathbb{R}_{++}^{l(S+1)}}$.

2) We calculate the individual composite mapping $g_{i}(s) \circ f_{i}(s)$ for all $s \in\{0,1, \cdots, S\}$ and show that $g_{i}(s) \circ f_{i}(s)=I d_{S \times \mathbb{R}++}^{(S+1)}$. This condition is satisfied since by definition of $g_{i}(s)$ we have

$$
\begin{aligned}
& f_{i}(s) \circ g_{i}(s) \\
= & g_{i}\left(\nabla u_{i}\left(f_{i}\left(p(s), w_{i}(s)\right)\right),\right. \\
& \left.\quad\left(f_{i}\left(p(s), w_{i}(s)\right)\right) \cdot \nabla u_{i}\left(f_{i}\left(p(s), w_{i}(s)\right)\right)\right) \\
= & \left(p(s), w_{i}(s)\right), \forall s \in\{0,1, \cdots, S\} .
\end{aligned}
$$

As required, we have established $f_{i}(s) \circ g_{i}(s)=I d_{S \times \mathbb{R}_{++}^{(S+1)}}$. We have proved the bijection property of the individual demand function ${ }^{4}$.

This proves existence of equilibria.

Definition 3. A feasible allocation $(x(s), y(s)) \in \mathbb{R}_{++}^{\operatorname{lm}(S+1)} \times \mathbb{R}^{\ln (S+1)}$ associate with equilibrium price vector $p(s) \in \mathbf{S}$ and economy $\omega(s) \in \mathbb{R}_{++}^{\operatorname{lm}(S+1)}$ is Pareto efficient for all $s \in\{0,1,2, \cdots, S\}$ if there is no other feasible allocation $(\bar{x}(s), \bar{y}(s)) \in \mathbb{R}_{++}^{\operatorname{lm}(S+1)} \times \mathbb{R}^{\ln (S+1)}$ such that for all $i \in\{1,2, \cdots, m\}$ and $s \in\{0,1,2, \cdots, S\}$

$$
u_{i}\left(\bar{x}_{i}(s)\right) \geq u_{i}\left(x_{i}(s)\right),
$$

with at least one strict inequality.

Theorem 6 (Pareto efficiency of model $\mathcal{P}(L)$ ). Every economy $\omega(s) \in \Omega$ of the model $\mathcal{P}(L)$ is Pareto efficient for all $s \in\{0,1, \cdots, S\}$.

Proof. We proceed by contradiction. We show that if at equilibrium price $p^{*}(s)$ the economy $\omega(x(s), y(s))$, where $(x(s), y(s))$ is an allocation of consumption and production which is not efficient, then it must be that firms do not maximize profits. This contradicts the assumption that all firms maximize profits (Debreu, [1] Chapter 5) and implies that not all economies are Pareto efficient.

We have for all $s \in\{0,1,2, \cdots, S\}$

$$
\sum_{j}^{n} \xi_{j}(p(s))=\sum_{j}^{n} \sum_{i}^{m} \theta_{i j}(s) \xi_{j}(p(s))=\sum_{i}^{m} \zeta_{i}(p(s)) .
$$

Hence,

\footnotetext{
${ }^{4}$ We have assumed that supply functions are smooth. Hence

$w_{i}(s)=p(s) \cdot \omega_{i}(s)+p(s) \cdot \zeta(p(s))$
}

$$
\begin{aligned}
& \sum_{i}^{m} f_{i}\left(p(s), p(s) \cdot\left(\omega_{i}(s)+\zeta_{i}(p(s))\right)\right. \\
= & \sum_{i}^{m} \omega_{i}(s)+\sum_{j}^{n} \xi_{j}(p(s)) .
\end{aligned}
$$

Hence we obtain the equilibrium equation given by

$$
\begin{aligned}
& \sum_{i}^{m} f_{i}\left(p(s), p(s) \cdot\left(\omega_{i}(s)+\zeta_{i}(p(s))\right)-\sum_{i}^{m} \xi_{i}(p(s))\right. \\
= & \sum_{i}^{m} \omega_{i}(s) .
\end{aligned}
$$

We can now establish a contradiction.

Now, let $p^{*}(s)$ be an equilibrium price vector for any arbitrary $\omega(s)$ and $(x(s), y(s))$ an associated feasible equilibrium allocation which is not Pareto efficient. Since $(x(s), y(s))$ is feasible we have

$$
\begin{aligned}
& \sum_{i}^{m} f_{i}\left(p(s), p(s) \cdot\left(\omega_{i}(s)+\zeta_{i}(p(s))\right)\right) \\
&= \sum_{j}^{n} \zeta_{j}(p(s))+\sum_{i}^{m} \omega_{i}(s) \\
& \sum_{i}^{m} f_{i}\left(p(s), w_{i}(s)\right)=\sum_{j}^{n} \zeta_{j}(p(s))+\sum_{i}^{m} \omega_{i}(s),
\end{aligned}
$$

hence

$$
\sum_{i}^{m} x_{i}(s)=\sum_{j}^{n} y_{j}(s)+\sum_{i}^{m} \omega_{i}(s) .
$$

Since by assumption $(x(s), y(s))$ is not Pareto efficient, there exists a feasible allocation $\left(\bar{x}_{i}(s), \bar{y}_{i}(s)\right)$ associated with with $\omega(s)$ and $p^{*}(s)$ such that

$$
u_{i}\left(\bar{x}_{i}(s)\right) \geq u_{i}\left(x_{i}(s)\right)
$$

with at least one strict inequality. This implies that

$$
p^{*}(s) \cdot \bar{x}_{i}(s) \geq p^{*}(s) \cdot x_{i}(s)
$$

with at least one strict inequality. Aggregating consumption bundles we obtain together with the inner product the strict inequality

$$
p^{*}(s) \sum_{i}^{m} \cdot \bar{x}_{i}(s)>p^{*}(s) \cdot \sum_{i}^{m} x_{i}(s) .
$$

Substituting Equation (5) into strict inequality (6) and using the feasible allocation $\left(\bar{x}_{i}(s), \bar{y}_{j}(s)\right)$ we obtain

$$
\begin{gathered}
p^{*}(s) \cdot\left(\sum_{j}^{n} \bar{y}_{j}(s)+\sum_{i}^{m} \omega_{i}(s)\right)> \\
p^{*}(s) \cdot\left(\sum_{j}^{n} y_{j}(s)+\sum_{i}^{m} \omega_{i}(s)\right) \\
p^{*}(s) \cdot \sum_{j}^{n} \bar{y}_{j}(s)>p^{*}(s) \cdot \sum_{j}^{n} y_{j}(s) .
\end{gathered}
$$


But this strict inequality says that for some $j \in\{1, \cdots, n\}$ that $p^{*}(s) \cdot \bar{y}_{j}(s)>p^{*}(s) \cdot y_{j}(s)$ for feasible $\bar{y}_{j}(s) \in Y_{j}(s)$. Hence a violation that firms maximize profits. Clearly, since $u_{i}\left(\bar{\omega}_{i}(s)\right)>u_{i}\left(\omega_{i}(s)\right)$ for at least one $i \in\{0,1, \cdots, m\}, \omega(\bar{x}(s), \bar{y}(s))$ is a Pareto inefficient economy.

Theorem 7. $\pi^{-1}(s)$ of model $\mathcal{P}(L)$ is a finite covering for every $\omega(s) \in \mathcal{R}$, for all $s \in\{0,1, \cdots, S\}$.

Proof. Let $\{p(s)\}$ consist of a single element of $\pi^{-1}(\omega(s))$ for all $s \in\{0,1, \cdots, S\}$. Consider the tangent map of elements of $\mathcal{E}$ not contained in the set of singular points, $p(s) \notin \mathcal{E}_{c}$. Then as a non singular point in $\mathcal{E}$ there exists a bijective map $D \pi_{p(s)}$ which by the inverse function theorem implies that $\pi(s): \mathcal{E} \rightarrow \Omega$ is locally a diffeomorphism. By the inverse function theorem there exists an open set $U(s)$ of $\omega \in \mathcal{R}$ and an open set $V(s)$ of $p(s) \in \mathcal{E}$ such that the restriction of the natural projection to $V(s),\left.\pi\right|_{V(s)}: V(s) \rightarrow U(s)$ is a diffeomorphism for all $s \in\{0,1, \cdots, S\}$. It follows from the one-to-one property of this map that $\pi^{-1}(\omega(s)) \cap V(s)=\{p(s)\}$. Since $V(s)$ is open in $\mathcal{E}$ it follows from the definition of open sets of $\pi^{-1}(p(s))$ as intersections with $\pi^{-1}(\omega)$ of open sets of $\mathcal{E}$ that the subset $\{p\}$ is open in $\pi^{-1}(p)$. The union of all open subsets $\{p(s)\} \in \pi^{-1}(\omega(s))$ define an open covering $\mathcal{P}$ of $\{p(s)\} \in \pi^{-1}(\omega(s))$. Compactness of the set $\pi^{-1}(\omega(s))$ follows from compactness of the preimage of a compact set $\{\omega(s)\}$ by the proper mapping $\pi(s): \mathcal{E} \rightarrow \Omega(s)$. It follows from compactness of $\pi^{-1}(\omega(s))$ that the open covering has a finite subcovering defined by the unique element of $\pi^{-1}(\omega(s))$. The union of a finite number of elements defines the set $\pi^{-1}(\omega(s))$ which is therefore a finite set. This proves finiteness of the number of equilibria.

\section{Conclusion}

This paper discusses local and global equilibrium properties of a production economy with a two period time structure and uncertainty. Adding uncertainty to the production model is a further step towards realism. It is shown that the equilibrium set of all production economies with uncertainty has the structure of a smooth submanifold of the Euclidean space which is diffeomorphic to a sphere. Beyond that, the paper shows that equilibria always exist, and that they are efficient and finite.

\section{REFERENCES}

[1] G. Debreu, "Theory of Value," New York, Wiley, 1959.

[2] G. D. K. Arrow, "Existence of an Equilibrium for a Competitive Economy," Econometrica, Vol. 22, No. 3, 1954, pp. 265-290.

[3] Y. Balasko, "Economic Equilibrium and Catastrophe Theory: An Introduction," Journal of Mathematical Economics, Vol. 46, No. 3, 1978, pp. 557-569.

[4] E. Dierker, "Topological Methods in Walrasian Economics," Vol. 92, Springer-Verlag, Berlin, 1974. doi:10.1007/978-3-642-65800-6

[5] Y. Balasko, "The Equilibrium Manifold: Postmodern Developments in the Theory of General Economic Equilibrium," The MIT Press, Cambridge, 1988.

[6] E. Jouini, "The Graph of the Walras Correspondence: The Production Economies Case," Journal of Mathematical Economics, Vol. 22, No. 2, 1993, pp. 139-147.

[7] G. Fuchs, "Private Ownership Economies with a Nite Number of Equilibria," Journal of Mathematical Economics, Vol. 1, No. 2, 1974, pp. 141-158.

[8] S. Smale, "Global Analysis and Econmics IV: Finitness and Stability with General Consumption Sets and Production," Journal of Mathematical Economics, Vol. 1, No. 2, 1974, pp. 107-117.

[9] T. Keho, “An Index Theorem for General Equilibrium Models with Production," Econometrica, Vol. 48, No. 5, 1980, pp. 1211-1232.

[10] T. Keho, "Regularity and Index Theoy for Econmies with Smooth Production Technologies," Econometrica, Vol. 51, No. 4, 1983, pp. 895-918.

[11] G. Debreu, "Smooth Preferences," Econometrica, Vol. 40, No. 4, 1972, pp. 603-615.

[12] K. Binmore, "Mathematical Analysis," 2nd Edition, Cambridge University Press, Melbourne, 1999.

[13] V. Guillemin and A. Pollack, "Differential Topology," Prentice Hall, Upper Saddle River, 1974.

[14] M. Hirsch, "Differential Topology," Springer, New York, 1972.

[15] J. Lee, "Introduction to Smooth Manifolds," Springer, New York, 2000. 